Voix et Images

volxetimages

\title{
L’Écriture : ultime recours, une entrevue avec André Major
}

\section{Jacques Pelletier, André Vanasse et Henri-Paul Jacques}

Volume 10, numéro 3, printemps 1985

André Major

URI : https://id.erudit.org/iderudit/200513ar

DOI : https://doi.org/10.7202/200513ar

Aller au sommaire du numéro

Éditeur(s)

Université du Québec à Montréal

ISSN

0318-9201 (imprimé)

1705-933X (numérique)

Découvrir la revue

Citer ce document

Pelletier, J., Vanasse, A. \& Jacques, H.-P. (1985). L'Écriture : ultime recours, une entrevue avec André Major. Voix et Images, 10(3), 12-21.

https://doi.org/10.7202/200513ar d'utilisation que vous pouvez consulter en ligne.

https://apropos.erudit.org/fr/usagers/politique-dutilisation/ 


\title{
L'Écriture: ultime recours, une entrevue avec André Major
}

\author{
par Jacques Pelletier, André Vanasse et la collaboration \\ d'Henri-Paul Jacques, Université du Québec à Montréal
}

V. et I. - Question générale pour commencer: LeFroid se meurt date de 1961, c'est-à-dire de pas loin de vingt-cinq ans. Quel bilan feriezvous de ces vingt-cinq ans d'écriture?

A.M. - Disons que j'ai l'impression d'avoir fait, durant une bonne partie de ce quart de siècle, un long apprentissage. De la langue, bien sûr, mais aussi de la vie. Ce n'est pas avant l'Épouvantail, c'est-à-dire au début des années 70 , que j'ai eu le sentiment de jouir d'une sorte d'autonomie créatrice. Auparavant, c'était moi qui écrivais sans doute, mais un moi sous influence, si vous voulez, encore à la recherche d'une identité, encore entravé par les modèles grâce auxquels, d'un autre côté, j'arrivais à voir un peu de quoi j'étais fait comme écrivain. Dans le Cabochon et la Chair de poule, j'ai délimité mon territoire; dans le Vent du diable, paru trois ans plus tard, $\mathrm{j}$ 'hésitais encore entre des tendances contradictoires, comme en fait foi le Carnet bleu qui fait suite au récit en le contestant. Pour me résumer, je dirais qu'avant les Histoires de déserteurs, j'ai surtout fait des essais de voix, des vocalises. Il m'a fallu tout un travail d'écriture et de remise en question de ce travail avant de comprendre qu'il fallait à tout prix éviter de coller au vécu immédiat et que le recul était indispensable à la création. Quand j'ai entrepris l'Épouvantail, vers 1971, j'ai utilisé une matière intime mais déjà lointaine souvenirs de famille, images et rêves qui constituaient ce que j'appellerais une mythologie fondatrice. Quand je dis rêves, je veux dire cauchemars.

V. et I. Qu'entendez-vous par cauchemars?

A.M. Des rêves de violence, de poursuite et d'assassinat. Mes nuits ressemblaient beaucoup a des séries noires. Ce n'est pas par hasard si je suis un dévoreur de littérature policière. Mais ce qui est curieux, c'est qu'au terme de la trilogie, je me suis trouvé délivré de ces fantasmes de violence, comme si je m'en étais purgé en les réduisant en mots. D'ailleurs, ce qui pousse à écrire, la plupart du temps, c'est le caractère obsessionnel de certaines images ou situations qui nous travaillent. J'ai fini par croire que toute cette violence me venait de l'espèce de terreur dans laquelle j'ai vécu durant mon enfance. Dans mon quartier, si on ne faisait pas partie d'une bande, on risquait de se faire casser la gueule en allant ou en revenant de l'école. À la maison aussi il fallait être sur ses gardes parce que ma mère recourait aux châtiments corporels pour nous dresser, comme elle disait. 
V. et I. - Le Carnet bleu qui fait suite au Vent du diable est-il vraiment autobiographique?

A.M. - Oui, délibérément autobiographique, comme pour faire contrepoids au récit qui le précède et qui me semblait piégé par mon parti pris fantastique. Il faut dire que j'ai écrit ce roman en même temps que mon étude sur Savard et que j'essayais à ce moment-là d'échapper au populisme de mes précédents livres. Sous l'influence de Savard, je voulais m'essayer au fantastique en utilisant des contes populaires, mais je me fourvoyais et le récit s'est finalement compliqué d'une intrigue amoureuse que je vivais alors tant bien que mal. Je n'arrivais pas à choisir entre le point de départ fantastique et le dérapage autobiographique, sauf dans ce Carnet bleu qui indique une volonté très nette de rompre avec le lyrisme naturaliste et sentimental du récit. Mais ce roman qui joue sur deux tons annonçait en quelque sorte les Histoires de déserteurs où s'opère la fusion entre la dimension fictive et la source autobiographique. En ce sens-là, le Vent $d u$ diable est un roman de transition en même temps que la liquidation du lyrisme.

V. et I. - Vous qui, sur un mode souvent comique, avez parlé de confession, comment voyez-vous la relation entre l'autobiographie et la fiction? Peut-on évacuer l'autobiographie du texte romanesque?

A.M. - Comme je le disais au sujet du Vent du diable, j'ai essayé, mais sans y parvenir, de travailler sur du fictif pur, sur une matière qui n'aurait aucun lien, même indirect, avec mon expérience de la vie. C'est une question de tempérament et de sènsibilité. Tout dépend du rapport qu'on a avec le langage. Moi, en tout cas, pour que ça fonctionne, pour que le projet s'incarne dans le langage, il faut que je m'alimente à une matière que je sens, qui me touche de près, qui me force à m'exposer en quelque sorte. Même pour la nouvelle - genre qui s'inspire davantage de l'extérieur - je pars de choses vues qui m'ont frappé sans que je sache toujours pourquoi. En ce sens-là, tout est autobiographique sans l'être. Parce que même s'il prend appui ou se constitue à même l'intimité du narrateur, le langage tend à universaliser cette part singulière en donnant sa propre version des choses. Chose certaine, je n'imagine pas qu'il me soit possible d'évacuer cette intimité qui exige d'être prise en charge par les mots. Qui cherche une issue dans le langage.

V. et I. - Une certaine critique a tendance à retracer les éléments autobiographiques de l'oeuvre et à reconstruire une sorte de trame significative à partir de ces éléments-là: L'écrivain est-il d'accord avec une telle démarche?

A.M. - Moi, je crois plutôt à une critique du texte. Parce qu'en fin de compte les élẹments utilisés par l'écrivain importent peu. Je veux 
dire que c'est le résultat qui compte, ce qu'il fait des matériaux auxquels il a recours. Le lecteur n'a pas besoin de savoir d'où ça vient, si c'est plus ou moins autobiographique. Il arrive même que l'écrivain ne distingue plus très bien, après coup, la part du vécu et la part du fictif, tout se fondant dans l'unique vérité vraiment décisive, celle du langage.

V. et I. L'intérêt repose donc avant tout sur le traitement des données autobiographiques, c'est-à-dire sur la forme que l'écrivain leur donne. Parlez-nous de votre évolution, du Cabochon à la Folle d'Elvis.

A.M. - Le Cabochon et la Chair de poule sont des textes que j'ai écrits plutôt instinctivement, à partir d'expériences personnelles et d'influences littéraires encore toutes chaudes. Sans le recul qu'aujourd'hui je trouve indispensable. Ce que je reprocherais à ces livres-là, c'est moins leur populisme que le moralisme qui tentait de les justifier. Mon attitude esthétique se ressentait de mes intentions délibérées d'agir sur le lecteur, de le prendre à parti. Il y avait dans ce choix la volonté de culpabiliser le lecteur supposément bourgeois. Avec le Vent du diable, comme je l'ai dit, j'ai voulu me défaire de tout ça en recherchant cette fois dans le lyrisme naturaliste un accord formel avec l'espèce de mythologie que Savard, Giono et Ramuz m'avaient aidé à mettre à jour. Mais mon fond réaliste devait refaire surface plus tard dans l'Épouvantail sans parti pris idéologique conscient. Entretemps j'avais pris des leçons chez Flaubert, Faulkner et chez les Russes: Tolstoï, Gogol et Tchékhov. J'ai évolué, je crois, vers une plus grande transparence, vers une forme de discrétion narrative, persuadé que le narrateur n'a pas d'autre fonction que de faire voir et sentir au lieu de se mettre lui-même en vedette, comme on le fait tant dans notre littérature, l'une des plus narcissisques qui soient, $j$ 'en ai bien peur. Curieusement, cette contrainte que je m'imposais m'a donné le sentiment d'une plus grande aisance langagière, comme si en m'effaçant derrière l'écriture je laissais toute la place à l'imprévisible foisonnement de la fiction. Et même si je voyais bien de quoi était fait ce foisonnement, de quelle part intime, je ressentais une étrange sensation d'étrangeté, d'objectivité si on préfère, comme si je n'avais été rien d'autre que l'instrument de cette parole par laquelle se manifestait tout un monde inédit et familier en même temps. Pour me résumer, j'ai fini par faire mien le précepte de Tchékhov selon lequel il faut écrire de sang-froid, quand on a cessé d'être à la fois juge et partie, quand on a perdu toute complaisance pour soi-même. Cette évolution formelle correspondait donc à une évolution intérieure. Je suis passé d'une remise en cause du monde extérieur - à l'époque de Parti pris - à une remise en cause de ce que j'étais, moi, peut-être parce que j'avais constaté que c'est d'abord soi qu'on change en écrivant. Mais, 
de toute manière, j'ai renoncé aux intentions, aux visées idéologiques. Je me suis simplement efforcé de voir un peu clair, de comprendre et d'atteindre quelque chose d'innommé, qui m'échappe encore, une espèce de vérité toujours provisoire, un peu plus grande, j'espère, qu'elle n'était la veille.

V. et I. - $\grave{A}$ vous lire parallèlement avec vos camarades de Parti pris, Renaud, Girouard, on retrouve les mêmes motifs: une mise en cause des tabous sexuels, et un même type d'écriture joualisante, à des degrés variables, bien sûr. Aviez-vous une sorte de programme commun?

A.M. - Pas vraiment. Nous avions en commun certaines lectures: Céline, Jean-Jules Richard, Albert Laberge; et le même désir de montrer que le joual exprimait une aliénation socio-culturelle. Il $\mathrm{y}$ avait aussi une réaction contre une écriture assez peu accordée au milieu ambiant, trop portée à imiter des modèles étrangers, français surtout. Tout ça était dans l'air. Même avant de joindre la revue, Gérald Godin allait dans ce sens-là. Depuis on a marché à fond de train dans cette voie, jusqu'à la rendre impraticable.

V. et I. - Dans votre décision de prendre vos distances avec Parti pris, est-ce qu'il n'y avait pas le sentiment d'étouffer à l'intérieur d'une sorte de politburo?

A.M. - Pour moi, comme aussi pour André Brochu, c'est vite devenu étouffant d'essayer de me conformer à une certaine ligne de parti, de concevoir la littérature en termes de pratique révolutionnaire, au moment même où je tentais de me dégager de visées non-esthétiques. Dans mon cas, le malaise se doublait d'un sentiment d'infériorité à la fois sociale et intellectuelle. J'étais le plus pauvre de la bande, et le seul qui ne sortait pas de l'Université. Il m'arrivait souvent de me sentir de trop au milieu de ces camarades qui maîtrisaient le discours théorique et parlaient du peuple avec l'assurance de ceux qui savent, tandis que moi, redevenu simple manutentionnaire chez Dupuis et frères, je n'étais plus sûr de rien ni de moi. Je me suis évidemment senti très coupable de rompre avec la revue, tout comme je m'étais senti coupable de rompre avec mon milieu d'origine. Il m'a bien fallu accepter d'être un déclassé, étranger partout, jamais à l'aise en public, tout en essayant de jouer le jeu, quand il le faut, tant bien que mal. Mon expérience avec Parti pris m'a beaucoup appris sur les rapports entre les êtres et sur moi-même, ce qui ne m'a pas empêché par la suite de me faire enrôler, par exemple, dans l'aventure coopérative des Quinze, puis dans la mise sur pied de l'Uneq. Apprendre à dire non, c'est long, ça fait mal. Mais quand on y arrive, c'est extrêmement satisfaisant.

V. et I. - Vous avez quand même dû vous sentir prisonnier d'une certaine image qu'on avait de vous, l'image d'un écrivain très 


\section{engagé. Comment avez-vous vécu cela?}

A.M. - J'ai eu du mal à m'en défaire, de cette image, mais j'ai pris tous les moyens pour la détruire sitôt après avoir rompu avec Parti pris. Je suis même allé plus loin que nécessaire, surtout au cours de mon passage au Devoir, jouant à contredire ce que j'avais été jusque-là. Je me suis fait prendre à mon propre jeu, prenant plaisir à provoquer la gauche et à me conformer à l'image d'homme de droite qu'on se faisait de moi avec ma collaboration. Et puis, quand j'en ai eu assez, quand $j$ 'ai compris que je faisais fausse route encore une fois, $j$ 'ai choisi d'être le cavalier seul qu'au fond j'avais toujours été. Le plus drôle de tout ça, c'est que la plupart des gens ne se souviennent même pas que j'ai été au Devoir de 67 à 70.

V. et I. - Votre dernière publication, la Folle d'Elvis, remonte à près de quatre ans. Et vous écriviez récemment dans Liberté que l'écriture ne vous manquait pas vraiment. Est-ce là un renoncement à l'écriture?

A.M. - Cette attitude de renoncement n'est pas nouvelle. Chaque fois que je publie un livre, la tentation de tout laisser tomber me reprend. Ce n'est pas un métier pour moi, écrire. C'est une sorte de nécessité à laquelle je me soumets, un peu à contrecoeur, même si je finis par $y$ trouver une grande satisfaction, irremplaçable d'ailleurs. Mais je trouve ça frustrant d'avoir ce besoin-là quand ce serait tellement plus simple de vivre comme à peu près tout le monde, sans avoir l'impression de sacrifier la vie de famille qui compte au moins autant que la littérature. En tout cas pour moi. Je ne fais pas partie de ces monstres qui ramènent tout à leur carrière et se laissent dévorer par l'oeuvre. Il faut dire que je ne vois plus l'art comme un salut, comme un avatar du vieil absolu religieux, essayant au contraire de considérer l'acte d'écrire comme un ultime recours, quand le silence m'étouffe, quand je me sens obsédé, jour après jour, par une image, une situation, un personnage, un style même. Contrairement aux boulimiques de l'écriture qui șe noient dans les mots, j'aimerais pouvoir vivre sans cette constante rumination intérieure qui, parfois, m'empêche de jouir tout simplement de cette vie qui est tout pour moi qui n'ai aucun espoir de survie. J'y arrive durant quelques mois, et puis ça se remet à me ronger. Il y a aussi, dans le renoncement provisoire à l'écriture, la volonté de ne pas refaire ce qui a été fait. Après chaque livre, et particulièrement les 600 pages des Histoires de déserteurs, j'avais épuisé toutes mes réserves imaginaires et il m'a fallu attendre que ce fond se reconstitue - c'est la vie qui s'en charge - à la fois semblable et différent de ce qu'il était. De la même manière, après la Folle d'Elvis, je me suis senti vide comme le désert, sans doute parce que je m'étais purgé de l'espèce de nostalgie qui traverse tout le 
recueil, et je n'attendais plus rien de la vie ni de moi, me sentant détaché de tout. J'ai vécu dans ce désert-là assez longtemps, pas loin de deux ans, avant de me remettre au travail.

Ce qui est dur aussi dans l'écriture, c'est d'être constamment en train de parasiter l'entourage, de penser à telle ou telle scène, et finalement de vivre dans une réclusion assez étouffante. J'en viens parfois à me demander si écrire n'est pá une tentative suicidaire, si avec chaque livre je ne creuse pas un peu plus ma fosse, du seul fait de remettre en question les attitudes et les rares certitudes qui m'aident à vivre. Je me demande ce qui me restera au bout du compte si je continue comme ça à démolir les défenses dont je m'entoure. Mais ceci dit, je sais bien que je ne peux. pas prendre ma retraite, même si on me disait que mes lecteurs en ont assez. Ce Journal que je publie dans Liberté, je l'ai justement entrepris pour ne pas me laisser engloutir dans le silence. C'est un compromis, mais qui m'a ramené à la fiction je ne sais trop comment.

V. et I. - Si on revient aux Histoires de déserteurs, on a d'abord affaire avec l'Épouvantail à une histoire policière, mais qui se transforme dans l'Épidémie et les Rescapés en histoire familiale. Comment expliquer ce changement?

A.M. $\quad-$ D'abord par le fait que je déteste faire ce que j'ai déjà fait et puis parce que les circonstances dans lesquelles j'ai écrit la trilogie ont changé. En ce qui concerne l'Épouvantail, après avoir fait une première version en 70 , à Toulouse, j'ai mis le manuscrit de côté jusqu'à l'été 72 , moment où je l'ai repris mais cette fois en le destinant à la Série noire. Je l'ai écrit très vite, en deux semaines, sous la tente. Mais à la relecture ça m'a paru trop linéaire, trop sec aussi. Je me suis alors mis à jouer avec l'ordre des chapitres, en me servant de la technique du flash-back sans me rendre compte que cela m'était commandé par la psychologie même de Momo, mon personnage principal, celui à partir de qui tout se noue finalement. Puis $j$ 'ai fait une autre version avant celle que j'ai publiée en conservant toutefois l'atmosphère policière, bien que l'écriture, elle, ait adopté une modulation et un rythme faulknériens. Je savais déjà qu'il y aurait une suite, que d'autres personnages à peine entrevus joueraient un rôle plus important, en particulier l'inspecteur Therrien à qui j'avais fini par m'identifier un peu. Mais dans l'Épidémie le ton change, l'allure et l'atmosphère aussi. C'est une roman plus hivernal, si on veut, plus méditatif, axé sur le temps et la mort, alors que l'Épouvantail l'était sur l'espace et la recherche de l'identité. Et, finalement, dans les Rescapés, j'ai essayé de montrer comment les rapports entre les êtres évoluaient dans un univers dont la cohérence ancienne avait disparu. C'est Jean-Marie Poupart, un lecteur assez perspicace, qui m'a signalé la charge ironique qu'il y avait dans cette écriture plus dépouillée, débarrassée en tout 
cas d'une certaine complaisance descriptive que je reproche a certaines pages des deux premiers volets. Mais si l'approche stylistique varie, de manière délibérée, je n'ai pas vraiment projeté d'écrire une histoire familiale, comme vous dites. Cela s'est fait tout seul. Mon souci était d'abord stylistique. Et je suis toujours à la recherche d'un style. C'est le style qui porte tout. Après coup, le livre achevé, le sens de cette recherche m'apparaît plus ou moins clairement, la plupart du temps grâce à ces quelques lecteurs clairvoyants sans lesquels la poursuite de l'oeuvre serait plus aventureuse qu'elle ne l'est.

Je ne pourrais pas, actuellement en tout cas, m'attaquer à une entreprise comme les Histoires de déserteurs. Pas seulement parce que j'ai perdu le goût de ces projets à long terme, mais parce qu'ayant liquidé cet héritage familial qui m'obsédait, je me suis davantage tourné vers le présent, vers ce qui m'entoure, et que je perçọis par flashes, d'où ma préférence pour la nouvelle, le texte bref, concis, ramassé.

V. et I. - Vous avez commencé par la poésie, genre que vous avez abandonné par la suite. Mais n'y aurait-il pas une nostalgie de la poésie dans votre nouvelle technique d'écriture?

A.M. - J'ai renoncé à la poésie pour la simple raison qu'elle était associée chez moi à la croyance. Et je suis mort comme poète dès que j'ai cessé de croire qu'un homme nouveau pouvait naître d'une révolution. Je ne dis pas que la poésie est impossible sans référence à un absolu quelconque, à un espoir, mais que pour moi il en a été ainsi. Je lis d'ailleurs avec plaisir des poètes comme Jaccottet, Benn, Guillevic, Brault et Beaulieu. Mais je ne fonctionne plus en poète, bien que j'essaie de pratiquer une prose allant au coeur des choses. La prose me convient mieux parce qu'elle me donne une plus grande liberté de manoeuvre, sans m'obliger à évacuer ce que j'appellerais les déchets du quotidien. Et puis j'aime les ingrédients du romanesque: les dialogues, l'atmosphère, les détails très physiques, les odeurs et l'apparente banalité de ces petits riens qui sont tout, comme dit Scerbanenco.

V. et I. - Comment voyez-vous la nouvelle écriture telle qu'elle se manifeste à la NBJ et aux Herbes rouges?

A.M. - Je m'intéresse aux problèmes formels, mais pas de cette manière, ni avec cette obsession de faire moderne à tout prix qui finit par être un objectif en'soi. Je travaille le langage sans renoncer à ce que j'appellerais le sex-appeal du réalisme. Je reprocherais globalement à la nouvelle écriture un parti pris moderniste qui se pique d'être subversif mais qui piétine dans un enclos où plus grand-chose ne circule. Ce qui est le plus consternant, chez la plupart de ces écrivains, c'est un manque flagrant d'ouverture, un repli craintif sur des positions aussi momifiées, 
aussi codifiées que l'académisme dont ils se prétendent les contempteurs. Il y a là un problème de culture, il me semble, que révèle la survalorisation de l'écriture comme miroir d'elle-même et de rien d'autre, ou de certains thèmes passe-partout, comme la ville ou l'américanité. Ils sont rares, les écrivains chez qui l'oeuvre est la conjonction d'une écriture et d'une pensée. D'ailleurs leur place dans l'institution littéraire est bien plus grande que leur apport réel à la création. Ce qui peut s'expliquer assez facilement par le système actuel fondé sur la subvention et, disons-le, par un certain snobisme de la critique universitaire. Je suis probablement injuste à leur égard, mais je ne m'en fais pas trop, ce que peut dire un lone ranger comme moi ne pèse pas lourd dans la balance.

V. et I. - Même si vous avez beaucoup évolué idéologiquement, il y a toujours eu chez vous une constante, une sorte de foi, disons, en l'indépendance. L'avez-vous toujours?

A.M. - La foi, non. Je veux dire que je ne crois plus comme avant que l'indépendance se fera ni même qu'elle est la condition sine qua non de notre épanouissement collectif. Le référendum m'a fait comprendre une chose que j'avais constatée dans Menaud comme ailleurs, à savoir que la dynamique de notre nationalisme est essentiellement défensive. En tant que peuple, nous sommes prêts à faire un bout de chemin vers l'autonomie, mais pas jusqu'au point où ça risque d'inverser les rôles. Très vite nous nous sentons coupables de ne plus jouer le rôle traditionnel de victimes. Et, contrairement à un courant de pensée qui misait sur notre passage de la condition minoritaire à la condition majoritaire, la pensée des Québécois s'en tient à la simple affirmation de ses valeurs je dirais affectives, la langue surtout, et du respect de nos droits acquis. Malgré le fait que le Canada Bill nous reste à travers la gorge et que rien ne nous le fera probablement avaler tel quel, nous avons perdu pour le moment tout désir d'autonomie, désireux avant tout d'avoir la paix et de régler des problèmes plus personnels, l'avenir pas très gai qui attend nos enfants. Il faut dire que nous avons eu notre ration de politique ces dernières années. Et puis l'échec référendaire n'est certainement pas étranger à l'espèce de fatigue culturelle qui nous tombe dessus.

D'autre part, on change, chacun de son côté, on devient plus pragmatique et on cesse de valoriser certains concepts. Je serais même prêt, quant à moi, à marcher dans un fédéralisme à la suisse, un peu comme celui que Joe Clark avait commencé à définir avant de se faire avoir par la réincarnation irlandopopuliste de Trudeau. Ce qui compte, finalement, c'est que le Québec fonctionne conformément à ses aspirations, pas plus. La déclaration d'indépendance, je peux m'en passer, à la condition, bien sûr, que le Canada s'ouvre les yeux et l'esprit s'il en est en- 
core capable. La politique n'est qu'un moyen, on l'a oublié ici où le Parti québécois a été chargé d'une mission quasiment religieuse: sauver la nation, en administrant un peuple encore adolescent, souvent irresponsable et d'un égoïsme social rare. Le Parti québécois paie aujourd'hui pour avoir pris la relève de l'Église et avoir incarné une espérance collective démesurée. Que nos intellectuels s'en prennent à lui sans mettre en cause leur propre aveuglement ne cesse de m'étonner, comme si ce parti n'était pas sorti tout droit de leurs propres exigences passées et de leur manque de réalisme aussi. On peut aider un peuple à comprendre son destin, on ne peut pas lui en imposer un autre, simplement parce qu'il nous convient mieux. De peur de nuire à leur image de grands-prêtres voués au culte de la nation, nos intellectuels se contentent de dénoncer le virage fédéraliste de Lévesque. Pas une seconde de réflexion: tout de suite la condamnation qui vous fait bien voir. Ce genre de lâcheté court les couloirs de nos institutions de haut savoir et nos salles de rédaction. De toute manière, même si l'Etat québécois montrait les dents, il ne ferait plus peur au Canada anglais qui ne veut rien savoir, le rapport de force jouant en sa faveur. Le nationalisme québécois survivra, dans sa version défensive, et il rebondira dès qu'on le piquera là où ça fait mal. En attendant que le rêve de réconciliation nationale de Mulroney s'évapore avec les brumes outaouaises, la nostalgie d'un Québec libre hantera quelquesunes de nos soirées si nous ne trouvons rien de mieux à évoquer en cette période de quarantaine où la question nationale semble être entrée. Ceci dit, je me sens étranger au catastrophisme des nationalistes crispés. Nous avons survécu à bien pire. Et puis, advenant même que le Canada français y laisse sa peau, on peut se consoler à la pensée que le Canada anglais ne fera pas de vieux os, lui non plus, n'étant déjà plus que l'ombre de l'oncle Sam.

V. et I. - Reste que, historiquement, la période la plus féconde culturellement a coïncidé avec la montée du nationalisme. $\mathrm{Ce}$ dynamisme-là, on ne le sent plus.

A.M. - Et c'est normal. De toute manière, cette effervescence des années 60 ne pouvait pas durer, elle devait plafonner, et ce qu'on a actuellement ce sont les retombées de cette effervescence. On est en pleine normalisation, si on veut. Le rêve québécois, qui tendait au consensus, s'est effrité en mille éclats de rêves personnels. En littérature, c'est pareil: au lieu d'un courant dominant, on a des choses qui vont dans toutes les directions. La fatigue culturelle aidant, on risque d'analyser la situation d'un point de vue défaitiste. Faute d'avoir changé les règles du jeu politique, nous sommes peut-être en train de changer ce qui peut l'être à l'échelle individuelle, espérons-le en tout cas.

V. et I. - Pour en revenir à votre oeuvre, on a beaucoup trop insisté sur 
l'arrière-plan social, comme en fait foi la discussion entre François Ricard et Jacques Pelletier dans Liberté. Est-ce que ce ne serait pas le familial ou le conjugal qui est davantage questionné dans votre oeuvre?

A.M. - Je vais faire plaisir à tout le monde en disant que, pour moi, c'est dans les rapports entre les êtres, entre homme et femme notamment, que se répercutent le plus dramatiquement les mutations sociales. C'est évident, je l'admets, dans tout ce que j'écris. Tout tourne autour de ce qui se passe entre l'homme et la femme.

V. et I. - Ce qu'on peut se demander, d'autre part, c'est la raison qui vous pousse à privilégier la campagne, par exemple dans les Histoires de déserteurs. Vous êtes-vous interrogé sur le choix qui doit avoir des conséquences sur le plan de l'écriture?

A.M. - Je me suis interrogé là-dessus dans la mesure où ce choix semble intriguer certains lecteurs. Mais je refuse d'y voir un véritable problème. Quand je travaille à un roman, c'est vrai que je prends, comme on dit, la clé des champs, sans doute parce que j'ai besoin d'espace et, curieusement, d'un milieu restreint, plus facile à saisir, tandis que dans la nouvelle, si j'aborde un aspect particulier du réel, forcément fragmentaire, je reviens en ville tout naturellement. Ce n'est pas vraiment un choix, à vrai dire. Les choses se présentent ainsi. Je ne planifie pas un roman en m'enjoignant de le situer au coeur de la ville ou à Rosement, par exemple. C'est une situation ou un motif romanesque qui s'impose à moi, et le décor de même. Au temps où je sociologisais, où je croyais bêtement que le roman contemporain devait être urbain par définition, je me reprochais mes incursions dans le Nord. J'ai fini par admettre que la liberté de l'écrivain devait être géographique aussi. D'ailleurs, à la campagne, on retrouve les mêmes valeurs qu'en ville, à cause de la télé. Les couples ne durent pas beaucoup plus longtemps dans les Laurentides, ni la vie d'ailleurs.

Il faut dire que, dans mon imaginaire, Montréal c'est le quartier de mon enfance, situé entre Fullum et Papineau, borné au nord par la rue Sherbrooke et au sud par le fleuve, et dont le coeur était le Stade des Royaux. Ces rues-là, avec leurs ruelles, leurs maisons de brique aux escaliers gris et aux persiennes vertbouteille, je peux m'en servir à ma guise, comme je me sers d'un lac ou d'un bois. C'est dans l'enfance, de toute manière, qu'on puise, et la mienne s'est nourrie aussi bien du paysage laurentien que de l'est montréalais. Sans compter que je suis de la première génération citadine de la famille. Normal que $j$ 'aie des racines ailleurs que rue Ontario. 